\title{
Asymmetric Organocatalysis Meets Hypervalent lodine Chemistry for the $\alpha$-Functionalization of Carbonyl Compounds
}

\author{
Davinia Fernández González, Fides Benfatti and Jérôme Waser ${ }^{\star[a]}$
}

\author{
Dedication ((optional))
}

In the last decade, organocatalysis has rapidly evolved to a mature field in asymmetric synthesis, complementary to enzymatic and organometallic catalyses. ${ }^{[1]}$ Nowadays, organocatalyzed transformations enrich the arsenal of the synthetic chemists who aim at the enantioselective construction of complex molecules. ${ }^{[2]}$ Organocatalysis possesses the advantage of relying on straightforward experimental procedures and readily accessible reagents. In fact, organocatalysts are cheap, easily available, environmentally benign and stable towards air and moisture. To profit completely from these benefits, the choice of reagents and co-catalysts in organocatalytic processes should be limited accordingly to moisture-tolerant compounds which do not interfere with the catalyst, by either coordination or covalent bonding. In this perspective, hypervalent iodine (III) reagents are well-suited for application in organocatalysis, because of their stability to air and moisture, and their tolerance towards many organic functional groups. ${ }^{[3]}$ Furthermore, their use as electrophilic reagents or oxidants in bond-forming reactions usually yields iodoarenes as by-product, which are inert under organocatalytic conditions. On the other hand, the compatibility of a nucleophilic organocatalyst (i.e. a secondary amine) with electrophilic loxidant species represents a potential pitfall, which requires fine-tuning of the organoiodane (III) properties by careful design. Perhaps because of this challenge, the two emergent fields of organocatalysis and hypervalent iodine chemistry have evolved in parallel and met only sporadically in the last decade, although with outstanding outcomes.

In the present article, selected examples of asymmetric reactions employing an amine or an ammonium as organocatalyst together with hypervalent iodine reagents for the $\alpha$-functionalization of carbonyl compounds are highlighted. ${ }^{[4]}$ The astounding advances made in asymmetric catalysis with chiral hypervalent iodine compounds, used catalytically or stoichiometrically, will not be discussed herein. ${ }^{[5]}$

[a] Dr. D. Fernández González, Dr. F. Benfatti, Prof. Dr. J. Waser

Laboratory of Catalysis and Organic Synthesis

Ecole Polytechnique Fédérale de

Lausanne, EPFL SB ISIC LCSO

BCH 4306, 1015 Lausanne

(Switzerland)

Fax: (+41) 21-693-9700

E-mail: jerome.waser@epfl.ch

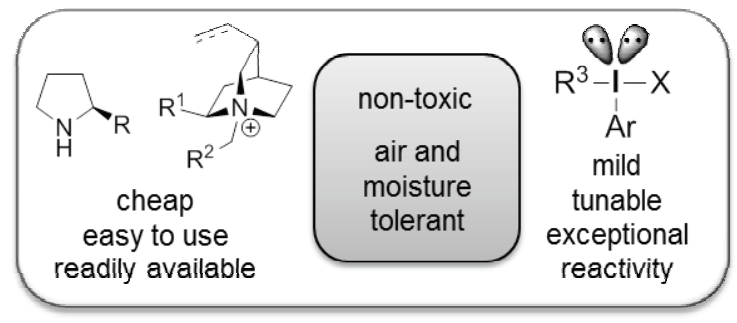

Figure 1. Advantages of organocatalysts and hypervalent iodine reagents.

The first asymmetric transformations exploiting hypervalent iodine reactivity in amine organocatalysis were oxidation reactions. In 2005, Córdova and co-workers described the use of iodosobenzene (1) as oxygen-transfer reagent in the $\alpha$ oxidation of cyclohexanones, catalyzed by (S)-proline (2) (Scheme 1). ${ }^{[6]}$ The protocol directly afforded $\alpha$-hydroxy cyclohexanones in moderate yields and enantioselectivities via enamine catalysis, whereas most other oxygen-transfer reactions using organocatalysts do not give the free alcohol as product. Nevertheless, a superior procedure for this asymmetric transformation employing molecular oxygen had been already reported by the same group,$^{[7]}$ which probably explains why no follow-ups of this reaction have appeared in the literature.

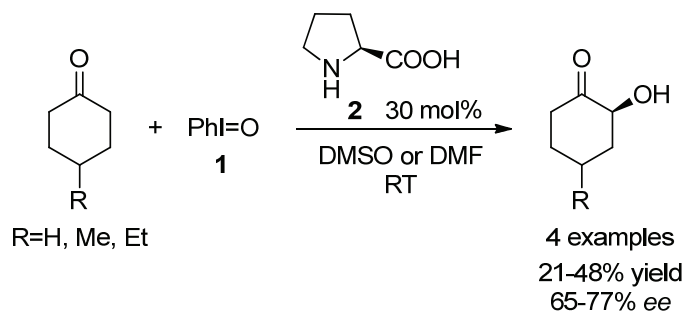

Scheme 1. Córdova's asymmetric organocatalytic $\alpha$-oxidation of ketones with iodosobenzene.

One year later, MacMillan and co-workers reported the organocatalytic asymmetric epoxidation of $\alpha, \beta$-unsaturated aldehydes using iodosobenzene as the oxygen source (Scheme 2) ${ }^{[8]}$ Intensive optimization of the reaction conditions was necessary to achieve excellent yields and 
enantioselectivities. Interestingly, the authors found that the controlled in-situ release of iodosobenzene via slow hydrolysis of [(nosylimino)iodo]benzene (3, NsNIPh) was crucial to avoid the catalyst oxidation. In this asymmetric epoxidation, imidazolidinone 4 was engaged in an iminium/enamine cascade, while iodosobenzene displayed an ambiphilic behaviour. However, as it was the case for the $\alpha$-hydroxylation reaction, numerous other organocatalytic methods are more efficient for the epoxidation of $\alpha, \beta$-unsaturated aldehydes. Consequently, these two early examples failed to reveal the real potential of hypervalent iodine reagents in organocatalysis.

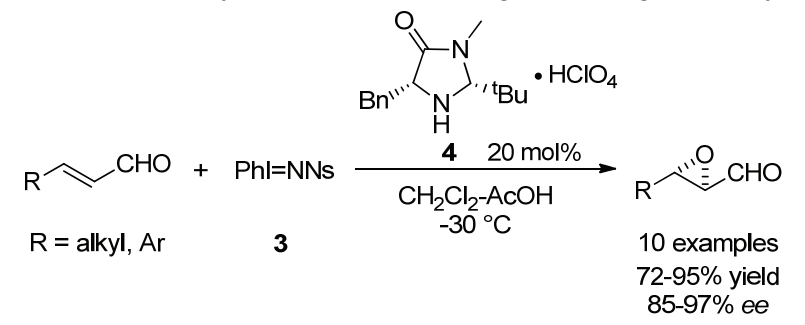

Scheme 2. MacMillan's asymmetric organocatalytic epoxidation using hypervalent iodine reagent (Ns = (4-nitrophenyl)sulfonyl).

A further proof of the compatibility of hypervalent iodine reagents with amine catalysis was given in 2007 by Gaunt and co-workers, who reported a beautiful enantioselective dearomatization-organocatalytic Michael addition cascade (Scheme 3). ${ }^{[9]}$ The careful choice of the oxidant (phenyliodonium diacetate, 5) was key for accomplishing a chemoselective oxidation of the phenol moiety to the quinone in the presence of the aldehyde and the amine. The best catalyst proved to be diarylprolinol $\mathbf{6}$, which allowed the asymmetric preparation of complex molecular architectures, starting from flat, aromatic molecules. In this example the group introduced in $\alpha$-position to the carbonyl did not originate directly from the hypervalent iodine reagent. Nevertheless, it proved for the first time that $\mathrm{C}-\mathrm{C}$ bond formation was possible in the presence of a hypervalent iodine oxidant, as previous methods used a stepwise oxidation-Michael addition approach.
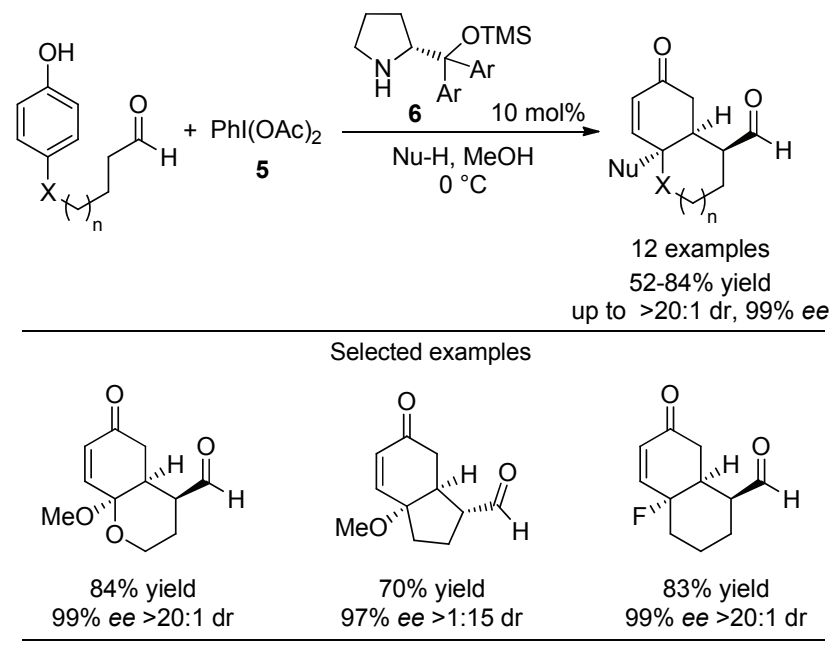

Scheme 3. Gaunt's enantioselective organocatalytic dearomatization. (TMS $=$ trimethylsilyl; $\mathrm{Ar}=2$-naphthyl).

Up to 2010 , there was no example of organocatalyzed C-C bond formation involving direct transfer of a substituent from a hypervalent iodine reagent. This is surprising, as nonasymmetric methods had been known for a long time. ${ }^{[4]}$ In
2010, MacMillan and Allen reported the first example of such a transformation, describing the enantioselective $\alpha$ trifluoromethylation of aldehydes (Scheme 4). ${ }^{[10]}$ In this case, the treatment of aldehydes with 3,3-dimethyl-1-trifluoromethyl1,2-benziodoxole (7) (Togni's reagent) in the presence of imidazolidinone catalyst 8 and $\mathrm{CuCl}$ as Lewis acid led to the formation of $\alpha$-trifluoromethylated products with high enantioselectivity. In this transformation, the potential of hypervalent iodine reagent in organocatalytic reactions is finally fully revealed. In fact, the only other reported organocatalytic method for the trifluoromethylation of aldehydes is based on photocatalysis and requires the use of difficult to handle gaseous $\mathrm{CF}_{3}$ [. $^{[11]}$

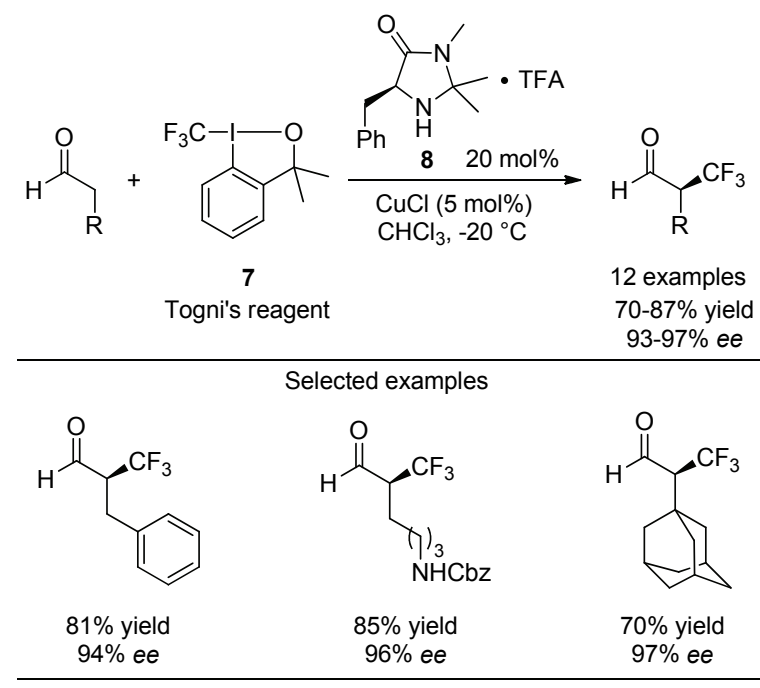

Scheme 4. Enantioselective $\alpha$-trifluoromethylation of aldehydes. (TFA = trifluoroacetic acid).

An important progress in the enantioselective $\alpha$ functionalization of aldehydes was reported in 2011 by MacMillan and co-workers. ${ }^{[12]}$ The non-toxic, readily accessible and air and moisture stable diphenyliodonium triflate salts $\mathbf{9}$ or 10 were used as arylating agents in an efficient organocatalytic reaction using chiral amine catalyst $\mathbf{1 1}$ in combination with $\mathrm{CuBr}$ as catalyst (Scheme 5). Importantly, organocatalytic $\alpha$ arylation of carbonyl compounds are difficult to achieve and had been limited to nucleophilic aromatic substitution of electron-poor aromatic compounds or addition to quinones. ${ }^{[13]}$ With the use of aryl iodonium salts, the transfer of nonactivated benzene rings became possible for the first time.

For both the arylation and the trifluoromethylation reactions, the use of a copper salt as co-catalyst was essential for an efficient reaction. The activation of the aldehydes proceeds most probably via the classical amine organocatalysis catalytic cycle involving an enamine A and an iminium B (Scheme 6). The role of the $\mathrm{Cu}$ catalyst was proposed to be activation of the electrophilic hypervalent iodine reagent (EI). Nevertheless, a different mechanism of activation was hypothesized for both transformations. In the case of 3,3-dimethyl-1-trifluoromethyl1,2-benziodoxole (7), $\mathrm{CuCl}$ probably acts as a Lewis acid to form a more electrophilic iodonium salt EI1. This intermediate is reactive enough to be attacked by the enamine $\mathbf{A}$, most probably via first $\mathrm{C}-\mathrm{I}$ bond formation to give $\mathbf{C}$, followed by a reductive elimination on iodine to give $\mathbf{B}$. In contrast, copper 
was proposed to be a redox active catalyst in the case of the aryl iodonium salt. Oxidative addition of $\mathrm{Cu}(\mathrm{I})$ on the hypervalent iodine reagent would lead to a highly electrophilic $\mathrm{Cu}$ (III) intermediate EI2, which would react with the enamine and lead to the arylation product after reductive elimination on copper via intermediate $\mathbf{D}$. These two reactions serve also well to remember the versatile nature of hypervalent iodine reagents, which can act either as electrophiles or oxidants and can even displayed "transition metal-like" properties.

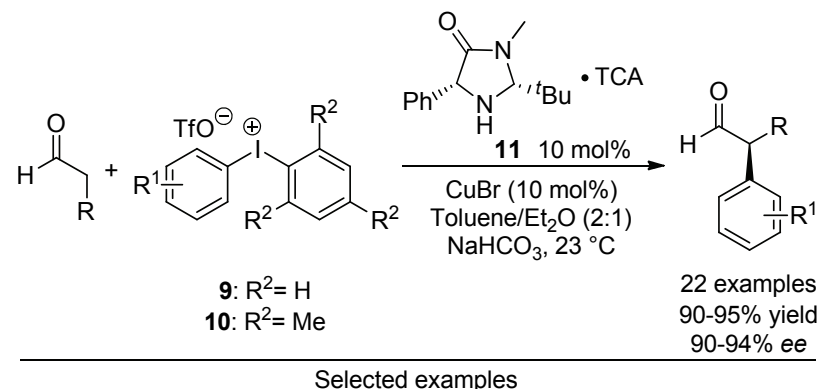

With 9;<smiles>CCCCCC(C=O)c1ccccc1</smiles>

$90 \%$ yield $92 \%$ ee

With 10;<smiles>O=CC(Cc1ccccc1)c1ccccc1</smiles>

\section{$87 \%$ yield}

$94 \%$ ee

$91 \%$ ee

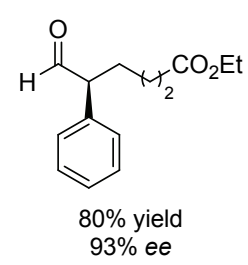

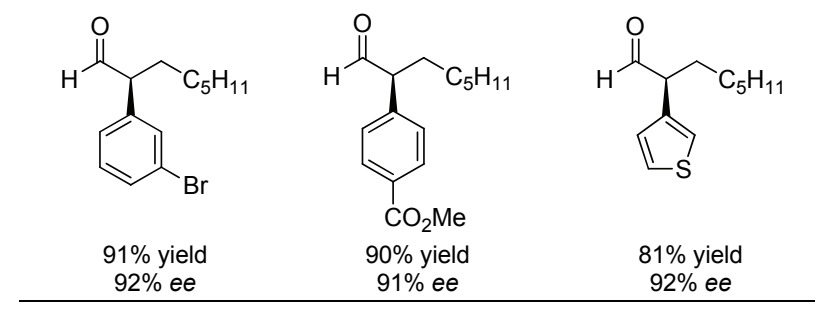

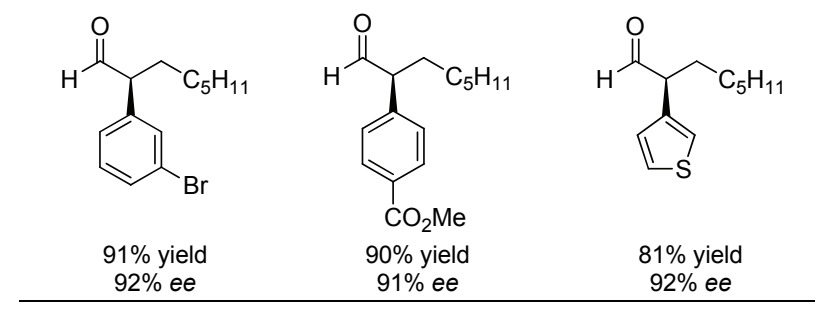

Scheme 5. Enantioselective $\alpha$-arylation of aldehydes. (TfO = trifluoromethanesulfonate; TCA = trichloroacetic acid).

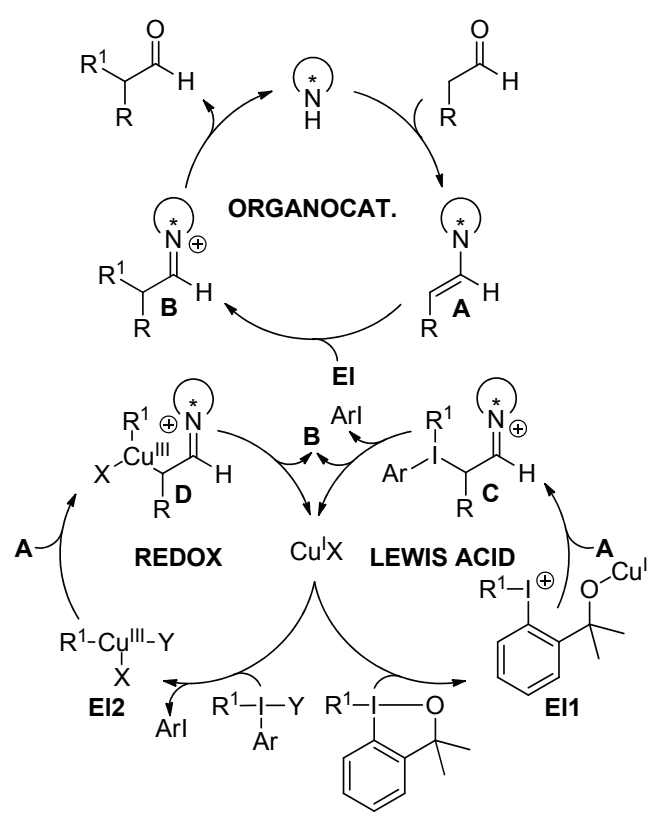

Scheme 6. Proposed mechanism for trifluoromethylation and arylation
In all the previous examples, the combination of organocatalysis and hypervalent iodine reagents for the $\alpha$ functionalization of carbonyl compounds has been based on enamine/iminium catalysis. Recently, both the arylation and alkynylation of ketoesters have become possible under phasetransfer conditions using hypervalent iodine reagent. ${ }^{[14]}$ In particular, our group reported the first example of asymmetric induction for the alkynylation of ketoesters using ethynylbenziodoxolone reagent 12 (TMS-EBX, Scheme 7). In this work, the use of a cinchona-derived ammonium catalyst 13 led to the formation of the free acetylene in $40 \%$ ee. $^{[15]}$ Although the observed enantioinduction is still too low to be preparatively useful, this result constitutes an important progress in the field, as the only other reported asymmetric alkynylation method using phase-transfer catalysts was limited to propiolate derivatives. ${ }^{[16]}$

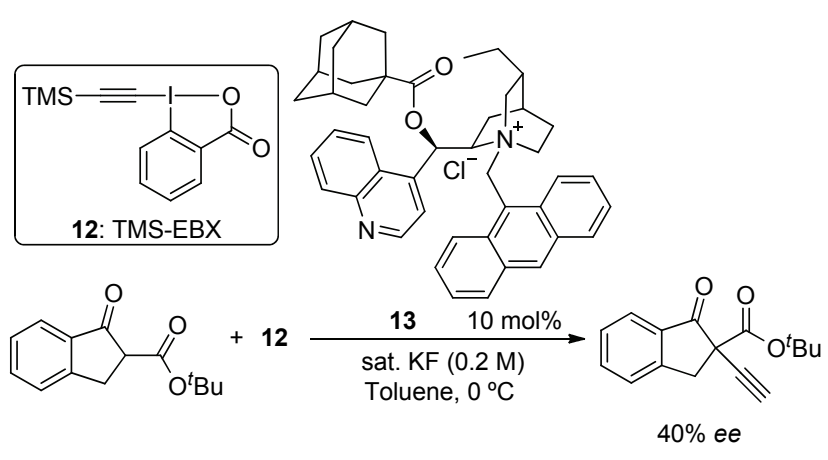

Scheme 7. Asymmetric $\alpha$-alkynylation of ketoesters.

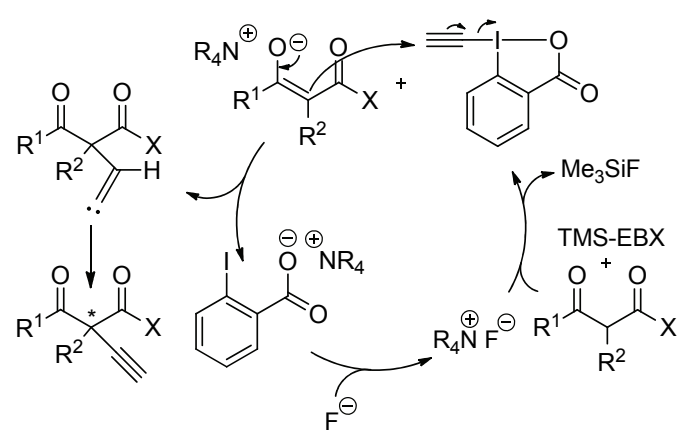

Scheme 8. Proposed Mechanism for the $\alpha$-alkynylation of ketoesters.

A possible mechanism for the $\alpha$-alkynylation of ketoesters using cinchona-derived ammonium catalyst is shown in Scheme 8 . The catalytic cycle is initiated by ammoniumcatalyzed transfer of the fluoride anion from aqueous to organic phase. The fluoride then deprotects the TMS-EBX reagent, generating a base able to deprotonate the ketoester to form an enolate intermediate bound to the chiral ammonium catalyst. Attack of the enolate on the $\beta$-position of the ethynyl benziodoxolone leads then to a carbene intermediate, which finally gives the alkynylated product via a Fritsch-ButtenbergWiechel rearrangement. Recently, Olofsson and co-workers have shown that a mechanism involving first attack of the oxygen of the enolate on the iodine followed by $\mathrm{C}-\mathrm{C}$ bond formation was favored in the case of aryliodonium salts. ${ }^{[14 b]}$ This result allowed them to rationalize why a racemic product was always obtained even when using chiral phase transfer catalysts, as the countercation is not bound anymore at the 
moment of $\mathrm{C}-\mathrm{C}$ bond formation. This alternative mechanism would also be possible for the alkynylation reaction. In this case however, the leaving carboxylate anion is covalently bond to the iodine, allowing asymmetric induction.

When considering these isolated but impressive results, it becomes apparent that the combination of organocatalysis and hypervalent iodine chemistry in enantioselective transformations is still largely underexplored. From this merger, new exciting synthetic possibilities are expected in the future, benefiting from the advantages of both areas.

\section{Acknowledgements ((optional))}

We thank the European Commission (Marie Curie IEF fellowship to F.B., grant number 253274) and the Swiss National Science Foundation (grant number 200021_119810/1) for financial support.

Keywords: organocatalysis • asymmetric synthesis hypervalent iodine $\cdot \alpha$-functionalization

[1] a) Asymmetric Organocatalysis: from Biomimetic Concepts to Applications in Asymmetric Synthesis, (Eds: A. Berkessel, H. Groeger), Wiley- $\mathrm{VCH}$, Weinheim, 2005. b) Enantioselective organocatalysis: reactions and experimental procedures, (Ed: P. I. Dalko), Wiley-VCH, Weinheim, 2007. c) Asymmetric Organocatalysis,(Ed: B. List); Top. Curr. Chem. 291; Springer: Berlin, Germany, 2010.

[2] Asymmetric Organocatalysis in Natural Product Syntheses, (Ed: M. Waser); Prog. Ch. Org. Nat. Prod. 96; Springer: Berlin, Germany, 2012

[3] a) Wirth, Hypervalent lodine Chemistry: Modern Developments in Organic Synthesis in Topics in Current Chemistry; Springer: New York, 2003; vol. 224. b) V. V. Zhdankin, P. J. Stang, Chem. Rev. 2008, 108, 5299-5358.

[4] As customary for a highlight, we will present in a concise way a subjective selection of recent examples. The interested reader is encouraged to consult the primary literature or the following comprehensive review on the general use of hypervalent iodine reagents for the $\alpha$-functionalization of carbonyls compounds: $E$. A. Merritt, B. Olofsson, Synthesis 2011, 517-538.

[5] For a few selected examples, see: a) T. Wirth, U. H. Hirt, Tetrahedron Asymmetry 1997, 8, 23-26. b) U. H. Hirt, B. Spingler, T. Wirth, J. Org.
Chem. 1998, 63, 7674-7679. c) U. H. Hirt, M. F. H. Schuster, A. N. French, O. G. Wiest, T. Wirth, Eur. J. Org. Chem. 2001, 1569-1579. d) R. D. Richardson, T. K. Page, S. Altermann, S. M. Paradine, A. N. French, T. Wirth, Synlett 2007, 538-542. e) S. M. Altermann, R. D. Richardson, T. K. Page, R. K. Schmidt, E. Holland, U. Mohammed, S. M. Paradine, A. N. French, C. Richter, A. M. Bahar, B. Witulski, T. Wirth, Eur. J. Org. Chem. 2008, 5315-5328, f) U. Faroog, S. Schafer, A. A. Shah, D. M. Freudendahl, T. Wirth, Synthesis 2010, 1023-1029. g) M. Ochiai, Y. Kitagawa, N. Takayama, Y. Takaoka, M. Shiro, J. Am. Chem. Soc. 1999, 121, 9233-9234. h) M. Fujita, S. Okuno, H. J. Lee, T. Sugimura, T. Okuyama, Tetrahedron Lett. 2007, 48, 8691-8694. i) J. Yu, J. Cui, X. S. Hou, S. S. Liu, W. C. Gao, S. Jiang, J. Tian, C. Zhang, Tetrahedron-Asymmetry 2011, 22, 2039-2055. For a review on the use of chiral hypervalent iodine reagents, see: j) $\mathrm{H}$. Liang, M. A. Ciufolini, Angew. Chem. Int. Ed. 2011, 50, 11849-11851. For a review on the catalytic generation of hypervalent iodine reagents and hypoiodites, see: k) M. Uyanik, K. Ishihara, ChemCatChem 2012, 4 177-185

[6] M. Engqvist, J. Casas, H. Sundén, I. Ibrahem, A. Córdova, Tetrahedron Lett. 2005, 46, 2053-2057.

[7] a) A. Córdova, H. Sundén, M. Engqvist, I. Ibrahem, J. Casas, J. Am Chem. Soc. 2004, 126, 8914-8915. b) H. Sundén, M. Engqvist, J. Casas, I. Ibrahem, A. Córdova, Angew. Chem. Int. Ed. 2004, 43 $6532-6535$.

[8] S. Lee, D. W. C. MacMillan, Tetrahedron 2006, 62, 11413-11424

[9] A.-N. Alba, M. Viciano, R. Rios, ChemCatChem 2009, 1, 437-439.

[10] A. E. Allen, D. W. C. MacMillan, J. Am. Chem. Soc. 2010, 132, 49864987.

[11] D. A. Nagib, M. E. Scott, D. W. C. MacMillan, J. Am. Chem. Soc. 2009, 131, 10875-10876.

[12] A. E. Allen, D. W. C. MacMillan, J. Am. Chem. Soc. 2011, 133, 42604263.

[13] For a few selected examples, see: a) M. Bella, S. Kobbelgaard, K. A Jorgensen, J. Am. Chem. Soc. 2005, 127, 3670-3671. b) J. Aleman, S. Cabrera, E. Maerten, J. Overgaard, K. A. Jorgensen, Angew. Chem., Int. Ed. 2007, 46, 5520-5523. c) J. Aleman, B. Richter, K. A. Jorgensen, Angew. Chem., Int. Ed. 2007, 46, 5515-5519. d) K. L. Jensen, P. T. Franke, L. T. Nielsen, K. Daasbjerg, K. A. Jorgensen, Angew. Chem. Int. Ed. 2010, 49, 129-133.

[14] a) D. Fernández González, J. P. Brand, J. Waser, Chem. Eur. J. 2010, 16, 9457-9461. b) P.-O. Norrby, T. B. Petersen, M. Bielawski, B. Olofsson, Chem. Eur. J. 2010, 16, 8251-8254.

[15] On-going investigations in our group have shown that optimization of the catalyst and ester structures can lead to a substantial increase in the enantioselectivity of the reaction (up to $79 \%$ ee): D. Fernández González, J. Waser, unpublished results.

[16] T. B. Poulsen, L. Bernardi, J. Aleman, J. Overgaard, K. A. Jorgensen, J. Am. Chem. Soc. 2007, 129, 441-449.

Received: ((will be filled in by the editorial staff))

Published online: ((will be filled in by the editorial staff)) 


\section{HIGHLIGHT}

Organocatalysis and hypervalent iodine (III) chemistry are coming increasingly in the focus of research. Interesting synthetic possibilities originate out of their merger, benefiting from the advantages of both areas. In the present highlight, selected examples combining asymmetric organocatalytic processes and hypervalent iodine reagents for the functionalization of carbonyl compounds are discussed.

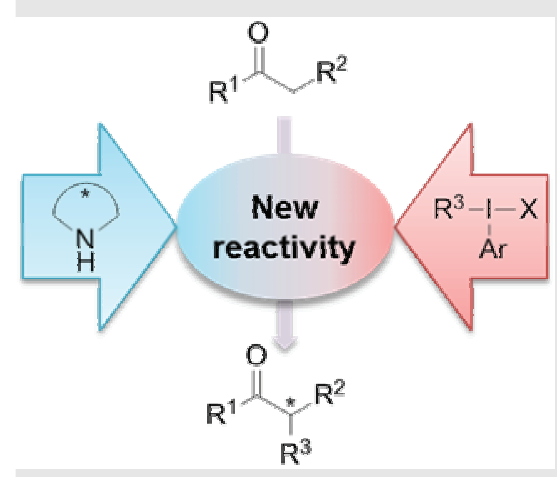

Davinia Fernández González, Fides Benfatti and Jérôme Waser*

Page No. - Page No.

Asymmetric Organocatalysis Meets Hypervalent lodine Chemistry for the $\alpha$-Functionalization of Carbonyl Compounds 